\title{
Nonlinear Dynamics Method for Excitation and Attenuation of Chandler Wobble
}

\author{
Wang Wen jun \\ Laboratory of Geodynamics, Institute of Geodesy and Geophysics \\ Chinese Academy of Science, Wuhan, CHINA, 430077 E-mail: \\ sq@asch.whigg.ac.cn
}

\begin{abstract}
In this paper, we introduce a nonlinear dynamical model for Chandler wobble including resonance excitation, bifurcation dissipation, frequency modulation, parameter resonance and visco-elastic long-time attenuation.
\end{abstract}

\section{Introduction}

Chandler wobble (CW) can be distinguished in polar motion observations. Its excitation and attenuation mechanism needs to be discussed in the case of viscoelasticity. Melchior (1957) discussed frequency modulation of the $\mathrm{CW}$. We obtained frequency modulation from the IERS data. Lambeck (1980) points out that excitation may be caused by unstable annual excitation, and that attenuation should be caused mainly by visco-elasticity. But, how and how much the CW is excited and attenuated by the annual excitation function, can be discussed according to the author's recent papers published in Chinese. Vondrák (1989) worked out the frequency instability of the CW and regarded it as an interesting problem. Gao Bu-xi (1993) obtained yearly CW amplitude and frequency by Fourier convolution, and provided amplitude modulation of the amplitudedependence. So, the instability of CW may be a key point for studying excitation and attenuation.

What is the reason for the instability in the CW? Wang (1998) raises a nonlinear resonance excitation mechanism, and points out that annual excitation provides energy transfer to the CW. Wang (1999a) obtains a main period solution and bifurcation of the $\mathrm{CW}$ in the nonlinear dynamical case, and points out that there may exist bifurcation dissipation. Later, the author (1999b) discusses the fact that frequency modulation of $3 \%$ may be the cause of an observed 18.6-year periodic variation. Then, $\mathrm{CW}$ frequency modulation of about 2-3\% may cause amplitude modulation on a scale of 40 years with an amplitude of 70\%. Recently the author showed that a sub-CW of 7 -month period may be excited by a nonlinear bifurcation from annual excitation.

In the past century, sufficient accurate geodesy for polar motion and many successful results for its mechanism have been obtained. Of course, in those physical factors observed by many advanced approaches, it has not been confirmed which one excites and damps the Chandler wobble and how. So, most geophysical mechanisms of polar motion are possible. Perhaps, the main reason why we couldn't confirm geophysical mechanisms is that the astro-geodynamics 
used all along has been only linear. If a little nonlinearity is introduced into the research, then the knowledge of polar motion would be improved.

\section{Resonance Excitation}

Physically in the Earth's rotation, there exists no motion of period 1.19 years identical with that of the CW. It may only be an annual excitation function that is close enough to transfer energy to the $\mathrm{CW}$. However, the frequency of the annual excitation is $f_{r}=1$, and its quality factor is about $Q_{r}=40$. For CW, $f_{c}=0.842, Q_{c}=50 \sim 70$, and the difference $\varepsilon=\left|f_{r}-f_{c}\right|=0.158$. Relatively, $\varepsilon / f_{c}=0.19$, so would annual excitation transfer energy to the $\mathrm{CW}$ resonance?

To begin, we consider the Earth's sway equations

$$
\begin{gathered}
Y^{\prime} / \sigma-X=-\psi_{1}, \\
X^{\prime} / \sigma+X=\psi_{2} .
\end{gathered}
$$

Under linear damping and forced sway model, adding linear damping in the $\mathrm{CW}$ differential equation and letting its damping attenuation index be $\lambda(>0)$, the dynamical equations become

$$
\begin{gathered}
X^{\prime}+\lambda X+\sigma Y=\sigma \psi_{2}, \\
Y^{\prime}+\lambda X-\sigma Y=-\sigma \psi_{1} .
\end{gathered}
$$

Distinguishing variables $\mathrm{X}$ and $\mathrm{Y}$,

$$
\ddot{X}+2 \lambda \dot{X}+\omega_{c}^{2} X=A_{r} \omega_{c} \cos \left(\omega_{r} t+\beta\right) .
$$

Noting that the ellipticity of the CW orbit is about 0.01 , the CW amplitude $\mathrm{U}$ can be approximated by a simple harmonic oscillation. So, Equation (3) represents the $\mathrm{CW}$ amplitude. The right hand side of (3) would come from annual excitation. Solving (3) by the complex amplitude method, it is easy to get the complex amplitude of the solution as:

$$
C=A_{r} \omega_{c} \frac{\left(\omega_{c}^{2}-\omega_{r}^{2}\right)-2 \imath \lambda \omega_{r}}{\left(\omega_{c}^{2}-\omega_{r}^{2}\right)+4 \lambda^{2} \omega_{r}^{2}} \cong a e^{\imath \delta} .
$$

Here, the real amplitude $a$ and phase $\delta$ have the form,

$$
a=\frac{A_{r} \omega_{c}}{\sqrt{\left(\omega_{c}^{2}-\omega_{r}^{2}\right)^{2}+4 \lambda^{2} \omega_{r}^{2}}}, \quad \tan \delta=\frac{2 \lambda \omega_{r}}{\omega_{c}^{2}-\omega_{r}^{2}},
$$

where, $a$ represents the contribution of resonance to $X$, and $\delta$ represents the phase delay after the occurrence of resonance. But, in fact, we set $\delta$ as positive by the relation $\tan \delta=\tan \left(\delta-180^{\circ}\right)$ and noting that the real phase delay should be $(\delta-180)$. By (9), it can be seen that, if $\omega_{r}=\omega_{c}$, then $\delta=90^{\circ}$, i.e. the phase would be $-90^{\circ}$; if $\omega_{r} \approx \omega_{c}$, and $\omega_{r}>\omega_{c}$, then $\tan \delta>0$, i.e., the real phase $\left(\delta-180^{\circ}\right)$ would be in the interval of $-90^{\circ}$ to $-180^{\circ}$. We can see that when $f_{r} \rightarrow f_{c}$ the damping attenuation index $\lambda$ tends to infinity by (4). 
Thus, in considering damping, the resonance excitation solution of $\mathrm{CW}$ should be of the form

$$
X=A_{c}^{\prime} e^{-\lambda t} \cos \left(\omega_{c} t+\phi^{\prime}\right)+a \cos \left(\omega_{r} t+\delta\right) .
$$

By (6), the frequency $\omega_{c}$ should decay to zero in its attenuation period, and the energy loss of overcoming the damping would need to be provided by resonance in the second term of the right hand side in (6). The energy which the $\mathrm{CW}$ absorbs from annual excitation by resonance in unit time is

$$
E=\frac{2 \lambda \dot{X} d X}{\omega_{c} d t}=\frac{2 \lambda \dot{X}^{2}}{\omega_{c}}=\frac{2 \lambda a^{2} \omega_{r}^{2} \sin \left(\omega_{r} t+\delta\right)}{\omega_{c}} .
$$

Letting $\varepsilon=f_{r}-f_{c}$, and averaging over time, we get the average transfer energy, i.e. the excitation energy from the annual excitation to the CW:

$$
E(\varepsilon)=\frac{A_{r} \omega_{c}}{4\left(\varepsilon^{2}+\lambda^{2}\right)} .
$$

Here terms smaller than $\varepsilon$ and $\lambda$ are deleted. Thus, the ratio which the annual excitation transfer to the $\mathrm{CW}$ by nonlinear resonance excitation is:

$$
\eta=\frac{E(\varepsilon)}{E(0)}=\frac{\lambda^{2}}{\varepsilon^{2}+\lambda^{2}} \times 100 \% .
$$

Estimating by (9), conserving CW energy, the rate of the transfer of energy from annual excitation to CW should be $\eta=9.1 \%$, where $\lambda=0.051, \varepsilon=0.161$. By this, it can be shown that more than $90 \%$ of the energy from the annual excitation function would excite annual polar motion and less than $10 \%$ of the energy would transfer to the CW.

If we take the $\mathrm{CW}$ inherent frequency $f_{c}=0.839$, average amplitude $A_{c}=$ 135 mas, and annual excitation amplitude $A_{r}=90$ mas, average phase variation from IERS polar motion data, or delay phase $\delta-180^{\circ}=113.4^{\circ}$, then we can inversely obtain from the upper formulations: average resonance forcing frequency $f_{c}=\bar{\gamma}=0.88$, average attenuation index $A_{c}=0.0426449$, average attenuation period $\tau=23.5 y r$, and average quality factor $Q=63$. Statistics in Table 1 provide important information on the $\mathrm{CW}$ variation.

\section{Theoretical Solution}

Annual excitation forcing frequency $\Omega$ would replace $\omega_{r}$ and the forcing phase $\phi$ would replace $\beta$. (3) would be easily solved by integration as (5) and (6) in Section 2 of this paper. However, the third term of the left-hand side of (3) should be replaced by a $\sin X$ term. Then, the CW should be considered as a nonlinear plane pendulum with linear damping, and periodic in the form

$$
\ddot{X}+2 \lambda \dot{X}+\omega_{c}^{2} \sin X=A_{r} \omega_{c} \cos (\Omega t+\phi) .
$$

$\sin X$ can be expanded into a Taylor series and approximated by adding a nonlinear term. We take one nonlinear term only in (10). Then the equation would be 
Table 1. Parameters for resonance excitation of Chandler wobble.

$\begin{array}{lllllll}\text { Term } & \text { Symb. } & \text { Mean } & \text { St.Var. } & \text { Min } & \text { Max } & \text { Appen. } \\ \text { CW Amplitude } & \text { Ac } & 132.5 & \mathbf{8 2 . 3} & 15.5 & 250 & 0.001 \\ \text { CW Frequency } & \text { fc } & .8425 & .027 & .77 & .87 & 1 / \text { yr } \\ \text { CW Period } & \text { Tc } & 433 & 13.56 & 474.3 & 417.4 & \text { day } \\ \text { Phase Difference } & \delta & 64.2 & 13.3 & 20.0 & 77.6 & \text { deg } \\ \text { Backward Phase } & \delta-180^{\circ} & -115.8 & & -160.0 & -102.4 & \text { deg } \\ \text { Resonance Frequency } & f_{r} & .8813 & .0608751 & .8301613 & 1.1239017 & 1 / \text { yr } \\ \text { Resonance Period } & T_{r} & 414.2 & 26.8 & 439.7 & 324.8 & \text { day } \\ \text { Outlier } & f_{r} 1937 & .8143491 & 1 / y r & \text { period: } & \mathbf{4 4 8 . 2 d a y ~} & \text { Maybe false } \\ \text { CW Relax.Expon. } & \lambda & .0414612 & .0242648 & .021482 & .108553 & 1 / \text { yr } \\ \text { CW Reson.Freq.Diff. } & \varepsilon & .0388 & .03446 & .006302 & .1934 & 1 / \text { yr } \\ \text { CW Freq.Qual.Factor } & Q c & 62.8 & 25.6 & 25 & 122 & \% \\ \text { CW Relax.Period } & \tau & 23.6 & 9.9 & 9.2 & 46.5 & \text { yr }\end{array}$

$$
\ddot{X}+2 \lambda \dot{X}+\omega_{c}^{2} X-\frac{1}{6} \omega_{c}^{2} X^{3}=A_{r} \omega_{c} \cos (\Omega t+\phi) .
$$

Usually, the $X^{3}$ term in (11) may be canceled. It is of the order of $10^{-13}$. On the contrary, in nonlinear theory, there are many cases in which a tiny nonlinearity can change the result. See Hao Bai-lin (1989).

Equation (11) is a standard Duffing one, and there are several standard methods of solution. Guckenheimer and Holmes (1983) surveys the Duffing equation in 2 dimensions thoroughly. We quote here the process of the perturbation method in one dimension, and then discuss amplitude and frequency in a 2-dimensional explanation of the $\mathrm{CW}$ solution.

The very last solution of (11) is

$$
X_{1}=A_{0} \cos \tau+\mu A_{1} \cos \tau+\frac{\beta^{\prime} A_{0}^{3}}{32 \omega_{c}^{2}} \cos 3 \tau+O\left(\mu^{2}\right)
$$

where

$$
\tau=\Omega t-\Phi_{0}-\mu \Phi_{1}-O\left(\mu^{2}\right) .
$$

In fact, (12) is a special solution of (11), and superposing the common solution and the special solution, we have, cutting off $O\left(\mu^{2}\right)$ terms,

$$
X=A_{0} \exp (-\lambda t) \cos \left(\omega_{0} t+\Phi_{0}\right)+\left(A_{0}+\mu A_{1}\right) \cos \tau+\frac{\beta A_{0}^{3}}{32 \omega_{0}^{2}} \cos 3 \tau
$$

As discussed in Section 2, the first term of the right hand side in (14) would die away by damping, so that the frequency $\omega_{c}$ would be quenched with time whereas a new phase (13) would alternate. This process is called module alternation. The new frequency $\Omega$ would be different and close to the quenching frequency $\omega_{c}$. $\Omega$ would replace $\omega_{c}$ as the inherent frequency and attenuate gradually and another new frequency would alternate.

Taking $F=A_{r} \omega_{c}$, say, $A_{r}=70$ mas, $\omega_{c}=0.84(2 \pi)$ and the initial value of $\Omega$ as $\Omega=\omega_{r}=\frac{1}{2 \pi}$, then three real (positive) roots of $\left(A_{0}\right)$ can be obtained and ignoring negative ones, we have: $A_{01}=135, A_{02}=36.839, A_{03}=105.037$. 


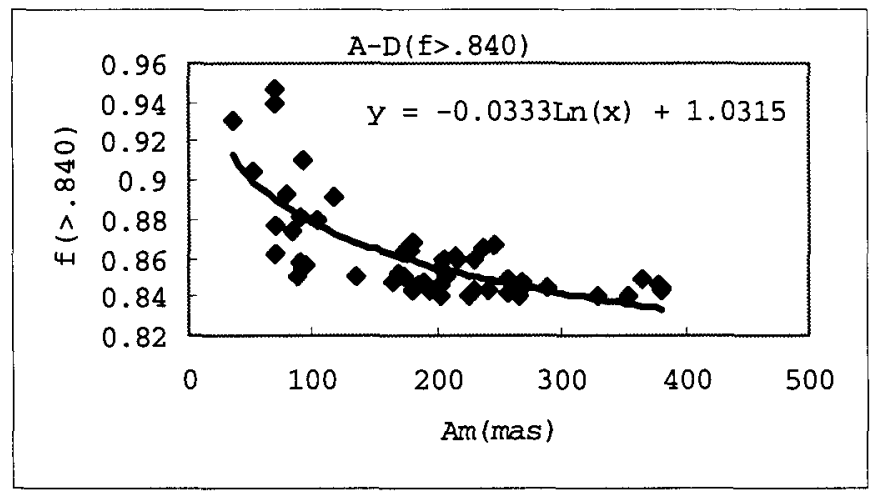

Figure 1. Resonance curve for $\mathrm{f}>0.84$.

Substituting into (14) and noticing that $\Phi_{0}$ should be negative: $\Phi_{01}=-113.87$, $\Phi_{02}=-90 \% 67, \Phi_{03}-93.03$.

For the first group of the solution, we find $A_{01}=135 \approx 132, \Phi_{01}=-113: 87$. This is the average $C W$ solution that we can usually obtain. $A_{01}$ exactly conforms to reality. On the other hand, we see in Section 1 that the backward phase caused by resonance excitation is $-113^{\circ} .4$, and $\delta=180^{\circ}-113^{\circ} 4=66^{\circ} .6$. It is interesting that, though we add a $X^{3}$ term in the equation, the result still holds and the backward phase calculated is $-113^{\circ}$ identical to the result without the $X^{3}$ term. Hence, this group of the solution is exactly the CW periodic one we always have known.

The second group of the solution is $A_{02}=36.839, \Phi_{02}=-90^{\circ} 67$. The third group is $A_{03}=105.037, \Phi_{03}=-93.04$. Since we have not solved the complex amplitude for the approximate Duffing equation, the respective frequencies of the two groups are not obtained directly. According to the number of solutions, it can be known that the two solutions would possess period-doubling and halfperiod respectively, i.e. the frequency of the second solution is $(1 / 2) \times 0.84 \times(2 \pi)$ whereas that of the third solution is $2 \times 0.84 \times(2 \pi)$. It is also interesting that the phase delays of the two solutions are nearly $-90^{\circ}$. Unfortunately, the two solutions are unstable, for the stability condition is not satisfied, while the unstable solution cannot be observed. Later in this paper, the stable bifurcation solutions are provided by the parametric resonance model.

In Gao (1993), an amplitude-dependence curve is obtained from the statistical result, and it is pointed out that, if frequency decreases, amplitude would increase and vice versa. However, we provide a resonance curve under the resonance excitation model of two branches, in which amplitude decreases if frequency decreases down to the inherent one 0.840 , while amplitude increases if frequency increases up to the inherent one. The result can be seen in Figure 1 and (15) and (16).

It can be said that the main energy of the CW is lost in overcoming the visco-elastic damping. As said in section 2 , it is illustrated by the $\mathrm{CW}$ solution (12) that almost all of the energy loss dissipated by visco-elastic damping is replenished by resonance excitation from the annual excitation of the $C W$. Also, 


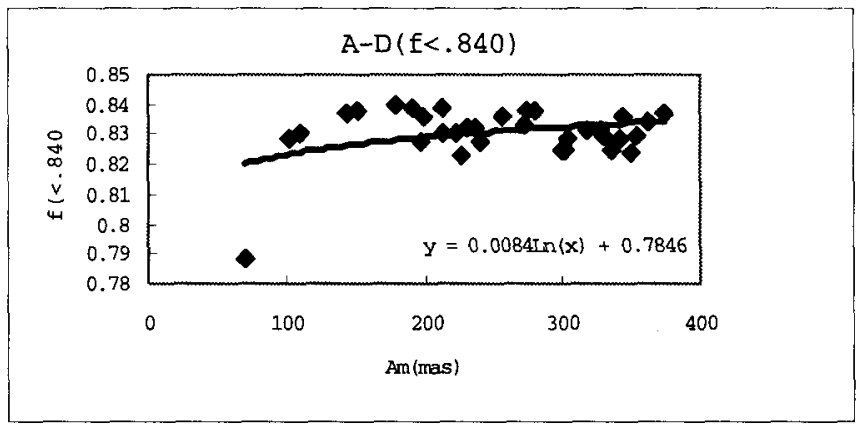

Figure 2. Resonance curve for $\mathrm{f}<0.84$.

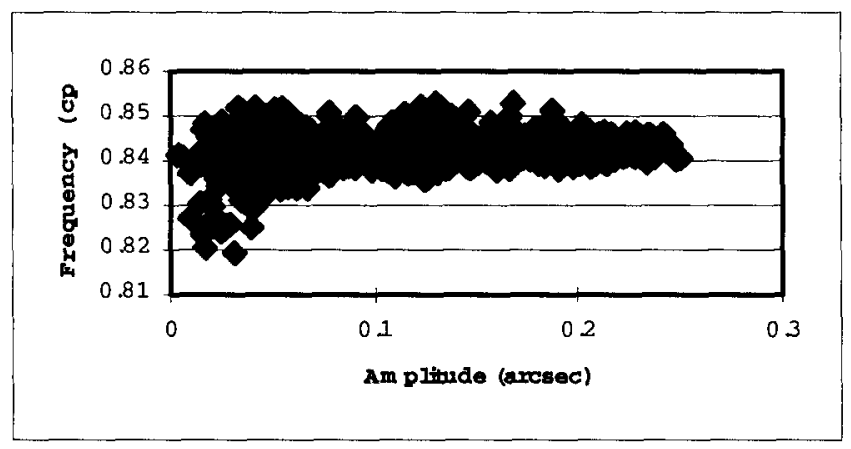

Figure 3. IERS amplitude-frequency graph.

we discuss the energy loss of the CW caused by nonlinear terms, and see that this part of the energy loss is evidently replenished by resonance excitation from an annual excitation too. However, there exists another part of energy loss shown in the solution called bifurcation dissipation.

$$
\begin{gathered}
f=-0.0333 \ln A_{m}+1.0315(f>0.840) \\
f=0.0084 \ln A_{m}+0.7846(f<0.840)
\end{gathered}
$$

It is the instability of phase resetting that produces the instability of the sway frequency, so that the energy transfer ratio $\eta$ of the resonance excitation fluctuates and makes the related stable annual excitation excite resonantly the unstable CW with a different frequency and variable amplitude. (5) shows the phase delay $\delta$ would be quite sensitive to frequency so that a minute variation of the frequency would cause a great deviation in $\delta$. In (7) $\delta$ appears in the form of $\tan \delta$, and $\tan \delta$ is more sensitive. Here it is evident that a minute nonlinearity would cause a huge variation. 
By analysis in section 2, it can be seen that a stable $\mathrm{CW}$ should have an average phase of about $-113^{\circ}$. If this value deviates only about $15^{\circ}$, the $\mathrm{CW}$ would preserve a regular sway. But as the deviation of the phase varies over $20^{\circ}$, we would see a phase loss event by which the CW could not have a regular amplitude any longer and the frequency moreover would not return to regularity for nearly 10 years. Now using ILS coefficients in Gao Bu-xi (1993), it can be seen that phase loss appears significantly from 1923 on. The phase loss for 21 years makes the CW amplitude drop to a minimum, of 35.5 mas in 1927 and the period to 385 days. But we can explain the resuming of the event by phase resetting. Accumulating the phase resetting series, we obtain the phase loss $-349^{\circ}$, i.e., in 21 more years, the total phase loss comes to about $-360^{\circ}$. By ( 7 ), it can be seen that there exists a function of period $180^{\circ}$, say, $\tan \delta$, so perhaps the minimum amplitude should resume in about 1930 where the accumulated phase loss came to $-180^{\circ}$. But, notice also (7) we can see that $\delta$ is a function of frequency of period $360^{\circ}$, then for amplitude and frequency to resume regular case, there should be accumulated a phase loss to $360^{\circ}$. Therefore, the $\mathrm{CW}$ resumed entirely by 1943 its amplitude after phase loss accumulated to $360^{\circ}$, so that there is no phase loss and minimum amplitude event in the coming years.

\section{Parameter Resonance}

There are evidently two peaks and three valleys, showing about a 32.5 -year average modulation. In Figure $4, Y_{a}$ shows a trend of equation $\mathrm{y}=3.5016 \mathrm{a}$ 3.4428. Subtracting the trend $Y_{a}$ is also seen to have a similar variation with a 32.5-year period.

The $\mathrm{CW}$ frequency is very unstable. It is not easy to observe perturbation in the CW phase variation time series. Okubo (1982) introduces a phase difference $\delta=(1 / f-1) \times 360^{\circ}$, which shows the phase difference directly relating to the reciprocal of the frequency. It modulates in a range of average $66^{\circ} 6$ (or $-113^{\circ} 4$ ). There are many more perturbation periods in the phase difference time series. An evident period is an 18.6-year period seen by spectral analysis. So, the phase difference has an 18.6-year perturbation period, and the frequency also has an 18.6-year perturbation period.

The perturbation of the CW amplitude is about 40 years. This 40 -year scale variation of CW has been noticed by many authors. Ye and Huang (1990) show that variation related to a 40-year scale in Earthquake rhythm (Zhu 1992). Here a possible analysis of this amplitude perturbation is provided.

As pointed out previously, $X_{a}$ and $Y_{a}$ are perturbed within a 32.5-year period, and the phase difference is perturbed within an 18.6-year period. The amplitude is related to positions and frequency so that its perturbation period would be the perturbation of 32.5-year and 18.6-year, i.e. it would be the minimum common multiple of 32.5 and 18.6. The minimum common multiple is only defined in integer numbers. However, Wang (1998) provides a generalized expression for the minimum common multiple. As [32.5/18.6]=1, where [n] is defined as the integer part of $\mathrm{n}$, then the period of amplitude Am is $T=$ $32.5 \times 18.6 /(32.5-18.6)=43.5439$ years.

The CW frequency time series is very stable, but possesses a correlation coefficient less than 0.08. Melchior (1957) obtains a frequency modulation of 


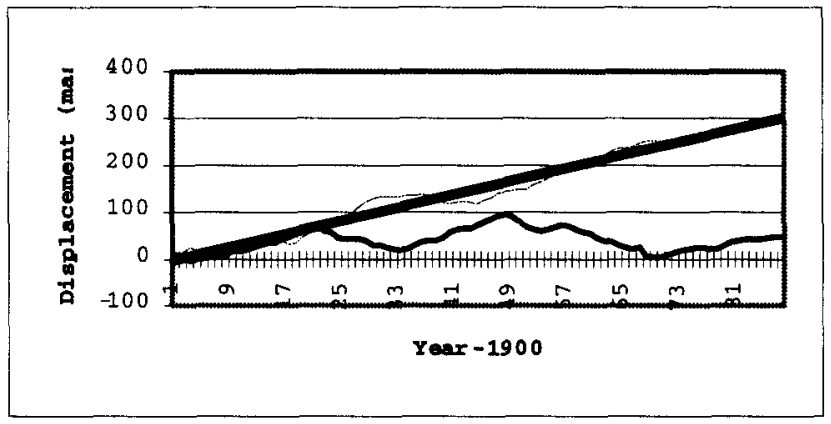

Figure 4. $\mathrm{x}$ and $\mathrm{y}$ time series.

about $4 \%$ with the ILS data. Okubo (1982) finds frequency modulation of about $1.4 \%$. Thus, there is no frequency modulation. Here we find a frequency modulation of about $2.9 \%$ with the IERS data. Furthermore, from a spectrum of $\mathrm{CW}$ frequency time series using pseudo-period analysis we detect an $\sim 18.6$-year pseudo-period, i.e. a period of not very significant detection.

By the relation between frequency and phase difference, we investigate the phase difference $\delta$. There is an 18.6-year period in the $\delta$ time series. This shows that the $\mathrm{CW}$ frequency is modulated by the generalized minimum common multiple in Okubo (1982). The modulation of the position axis with a 32.5 year period can be seen as a frequency resonance of period 1.193 year and 18.6 years and 8.85 years. The perturbation period of the $\mathrm{CW}$ amplitude would be the resonance period of 32.5 years and 18.6 years. It is almost entirely identical with the previous estimation. Here we investigate what amount of perturbation would occur in the $\mathrm{CW}$ amplitude associated with $2.9 \%$ frequency modulation. As the two parameters are perturbed at the same time, $\mathrm{CW}$ is a so-called parameter resonance. The main condition of resonance is that the real frequency $\Omega$ of the system is close to that of the perturbation frequency $\omega_{o}$ of $\lambda$ and $\omega_{c}$.

The parameter resonance model appears as a nonlinear equation. The $\mathrm{CW}$ amplitude would satisfy a variable coefficient nonlinear differential equation. Let $\lambda_{o}$ be the perturbation amount of attenuation index, $\Delta \omega$ be that of frequency, $\omega_{o}$ the modulation frequency of $\omega_{c}$ and $\lambda, \lambda$ the average attenuation index, $A_{r}$ the annual excitation amplitude, and $\Omega$ the forcing frequency.

$$
\begin{gathered}
\frac{d^{2} X}{d t^{2}}+\alpha(t) \frac{d X}{d t}+\beta(t) X=\gamma(t), \\
\alpha(t)=2 \lambda(t)=2 \lambda+2 \lambda_{0} \cos \omega_{0} t \beta(t)=\left(\omega_{c}+\Delta \omega \cos \omega_{0} t\right)^{2} .
\end{gathered}
$$

As the parameter is varying with time, a solution of the equation would be very difficult. Wang (1999c) gives a reasonable solution. Set $\omega_{c}=0.8425$, $\omega_{o}=\omega_{c} \pm \Delta \omega, \Delta \omega=1 / 43.5=0.0228, \lambda=0.04248, \lambda_{o}=0.021$. It gives an amplitude perturbation of 104.1 mas on the basis of the average CW amplitude of 132.5 mas for the ILS data. In fact, the real observation of the ILS has a minimum of 10-20 mas, and a maximum of 249.6 mas. In the parameter 
resonance solution of period, the phase difference varies from $-113.4^{\circ}$ to $-116.4^{\circ}$, and the period varies from 440.7 days to 429.3 days.

\section{Bifurcation solution of sub-CW}

Some authors have obtained sub-CW of 7-month period from the IERS data and estimated an amplitude of about 10 mas. However, there exists no excitation of 7 -months period in the atmosphere, ocean or others. Here, a possible mechanism of nonlinear dynamics is provided by a parameter resonance time dependence model. The solution of (3) in the condition of (18) is of the form

$$
a=a_{0} \exp \left\{F\left(\theta, \theta_{0}\right)\right\}
$$

where

$$
\begin{gathered}
\left(2 \theta_{0}\right)=\tan ^{-1}\left[\frac{\frac{1}{\omega_{0}+2}-\frac{1}{\omega_{0}-2}}{\frac{1}{\omega_{0}+2}+\frac{1}{\omega_{0}-2}} \sin \left(\omega_{0} \pi\right)\right], \\
a_{0}=\frac{(2 / \pi) A_{r} \cos \theta_{0}}{4\left|\sigma_{c}-\omega_{0}\right|-d_{2}^{(1)} \sin 2 \theta_{0}-d_{2}^{(2)} \cos 2 \theta_{0}} . \\
\text { Here, } d_{2}^{(1)}=\left[\frac{1}{\omega_{0}+2}+\frac{1}{\omega_{0}-2}\right]\left[\frac{2 \lambda_{0}}{\pi} \sin \left(\omega_{0} \pi\right)+\frac{\omega_{0}}{2 \pi}\left(1-\cos \left(2 \omega_{0} \pi\right)\right)\right]+O\left(10^{-6}\right), \\
d_{2}^{(2)}=\left[\frac{1}{\omega_{0}+2}+\frac{1}{\omega_{0}-2}\right]\left[\frac{2 \lambda_{0}}{\pi}\left(1-\cos \left(2 \omega_{0} \pi\right)\right)+\frac{\omega_{0}}{2 \pi}\left(\sin \left(\omega_{0} \pi\right)\right]+O\left(10^{-6}\right) .\right.
\end{gathered}
$$

Then, the maximum amplitude of sub-CW can be solved by replacing $\theta_{0}$ as $\pm \theta_{0} / 2$. If the solution is obtained by $+\theta_{0} / 2$, then sub-CW can be provided. $\mathrm{By}$ (20), it can be shown

$$
a_{0}=0.2929875 A_{r}=17.97 \approx 18(\text { mas }) .
$$

So, the sub-CW of 7-month period should possess a maximum amplitude of 18 mas. According to the solution (22), the sub-CW ought to have a minimum of 0 . Thus, we obtain the amplitude of the average sub-CW as about 9 mas. That is identical to the observational result given by some authors mentioned above.

Examining the stability condition of the bifurcation solution, it can be seen that the condition is satisfied. So, the sub-CW solution of 7 months is stable and can be observed. As to the double-period CW, it can be solved by replacing $\theta_{0}$ with $-\theta_{0} / 2$ and getting its maximum amplitude. But it is so weak that it would be lost in the CW and is not easily detected. Since the excitation of 7 -month period cannot be found in physical phenomena, the sub-CW may be caused by bifurcation of the annual excitation function. 


\section{Conclusion}

The amplitude and frequency of the CW are unstable or modulated by longterm astronomical forces. An amplitude dependence can be explained by a nonlinear dynamics model. Excitation of the $\mathrm{CW}$ can be obtained by a resonance excitation model and energy loss by visco-elasticity is replenished by an annual excitation function. Supposing that the frequency of the $\mathrm{CW}$ is modulated as observed by the frequency time series, according to a parameter resonance model, the amplitude of the CW would be modulated by about $20 \% \sim 180 \%$ of the average $\mathrm{CW}$ - identical to observations. By the parameter resonance model, the sub-CW can be found to be 9 mas with a period of 7 months, so the sub-CW is only excited by nonlinear bifurcation from the annual excitation function.

\section{References}

Gao Bu-xi, 1993, Science in China (Series B) 23, 553.

Guckenheimer, J. \& Holmes, P., 1983, Springer-Verlag.

Hao, B.L., 1989, World Scientific.

Lambeck, K., 1980, The Earth's Variable Rotation: Geophysical Causes and Consequences, Cambridge Univ. Press, Cambridge.

Melchior, P., 1957, Latitude Variation, Progress in Physics and Chemistry of the Earth, Pergamon Press.

Okubo, S., 1982, Geophys. J. R. astr. Soc., 71, 647.

Vondrák, J., 1989, Report No. 402, The Ohio Univ., Columbus.

Wang Wen-jun, 1998, Acta Astronomica Sinica, 39, 287.

Wang Wen-jun, 1999a, Acta Astronomica Sinica, 40, 94.

Wang Wen-jun, 1999b, J. Of Wuhan Technical University of Mapping and Surveying, 3.

Wang Wen-jun, J. 1999c, Nonlinear Dynamics in Science and Technology, 6, 60. Ye Shu-hua and Huang Cheng, 1995, Scientific Introduction, 1.

Zhu Yao-zhong, 1992, Acta Astronomica Sinica, 33, 420. 